University of Wollongong

Research Online

Faculty of Engineering and Information

Faculty of Engineering and Information

Sciences - Papers: Part A

Sciences

2013

Online in vivo dosimetry in high dose rate prostate brchytherapy with MOSkin detectors: In phantom feasibility study

\author{
G Gambarini \\ Universita degli Studi di Milano-Bicocca \\ M Carrara \\ Fondazione Irccs Istituto Nazionale Tumori \\ C Tenconi \\ Universita degli Studi di Milano-Bicocca \\ N Mantaut \\ Universita degli Studi di Milano-Bicocca \\ M Borroni \\ Fondazione Irccs Istituto Nazionale Tumori
}

See next page for additional authors

Follow this and additional works at: https://ro.uow.edu.au/eispapers

Part of the Engineering Commons, and the Science and Technology Studies Commons

Research Online is the open access institutional repository for the University of Wollongong. For further information contact the UOW Library: research-pubs@uow.edu.au 


\title{
Online in vivo dosimetry in high dose rate prostate brchytherapy with MOSkin detectors: In phantom feasibility study
}

\begin{abstract}
MOSkin detectors were studied to perform real-time in vivo dose measurements in high dose rate prostate brachytherapy. Measurements were performed inside an urethral catheter in a gel phantom simulating a real prostate implant. Measured and expected doses were compared and the discrepancy was found to be within $8.9 \%$ and $3.8 \%$ for single MOSkin and dual-MOSkin configurations, respectively. Results show that dual-MOSkin detectors can be profitably adopted in prostate brachytherapy treatments to perform real-time in vivo dosimetry inside the urethra.
\end{abstract}

\section{Keywords}

high, dose, rate, prostate, online, brchytherapy, vivo, moskin, detectors, phantom, feasibility, study, dosimetry

Disciplines

Engineering | Science and Technology Studies

\section{Publication Details}

Gambarini, G., Carrara, M., Tenconi, C., Mantaut, N., Borroni, M., Cutajar, D., Petasecca, M., Fuduli, I., Lerch, M., Pignoli, E. \& Rosenfeld, A. (2014). Online in vivo dosimetry in high dose rate prostate brchytherapy with MOSkin detectors: In phantom feasibility study. Applied Radiation and Isotopes, 83 (Part C), 222-226.

\section{Authors}

G Gambarini, M Carrara, C Tenconi, N Mantaut, M Borroni, D Cutajar, M Petasecca, I Fuduli, M Lerch, E Pignoli, and Anatoly B. Rosenfeld 
Online in vivo dosimetry in high dose rate prostate brchytherapy with MOSkin detectors: in phantom feasibility study

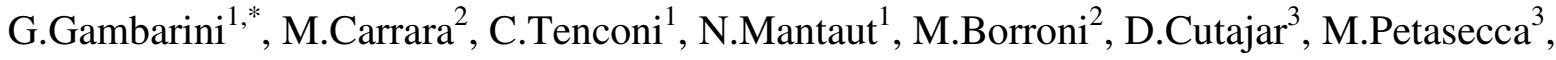 \\ I.Fuduli $^{3}$, M.Lerch $^{3}$, E.Pignoli ${ }^{2}$, A.Rosenfeld ${ }^{3}$ \\ ${ }^{1}$ Department of Physics, Università degli Studi di Milano, and INFN, Milan, Italy \\ ${ }^{2}$ Medical Physics Unit, Fondazione IRCCS Istituto Nazionale Tumori, Milan, Italy \\ ${ }^{3}$ Centre for Medical Radiation Physics, University of Wollongong, Wollongong, NSW, Australia
}

* Corresponding Author

Grazia Gambarini

Department of Physics

Università degli Studi di Milano and INFN

Via Celoria 14

I-20133 Milan, Italy

\title{
Highlights
}

- A needles implant was set up in phantom to simulate prostate brachytherapy treatments

- In vivo dosimetry was performed in the urethral catheter with MOSkin dosimeters

- Dual-MOSkin detectors resulted to be accurate dosimeters to perform this task 


\begin{abstract}
MOSkin detectors were studied to perform real-time in vivo dose measurements in high dose rate prostate brachytherapy. Measurements were performed inside an urethral catheter in a gel phantom simulating a real prostate implant. Measured and expected doses were compared and the discrepancy was found to be within $8.9 \%$ and $3.8 \%$ for single MOSkin and dual-MOSkin configurations, respectively. Results show that dual-MOSkin detectors can be profitably adopted in prostate brachytherapy treatments to perform real-time in vivo dosimetry inside the urethra.
\end{abstract}

\title{
Keywords
}

High dose rate, prostate brachytherapy , MOSFET, in vivo dosimetry 


\section{Introduction}

\subsection{In vivo dosimetry in radiotherapy}

The recent developments of more sophisticated radiotherapy and brachytherapy (BT) techniques call for the improvement of instruments and methodologies employed for the quality control of the performed treatments. Due to the achievable high conformity of modern BT associated with steep dose gradients, a careful verification of the accuracy in the delivered dose distributions, as planned by the Treatment Planning System (TPS) through mathematical models, is gaining importance.

In vivo dosimetry is a reliable method to compare planned and delivered dose distributions, representing therefore a valid tool to systematically verify treatment accuracy and improve radiotherapy quality control (Lambert et al 2007, Mijnheer 2008). Particularly advantageous for in vivo dosimetry are detectors that allow on-line dose reading. These dosimeters provide in fact realtime measurements during treatment, avoiding therapy misadministration and allowing at the same time intraoperative dose re-planning for treatment error correction.

Current methods for in vivo dosimetry are mainly based on the application of thermoluminescence detectors (TLDs) (Toye et al 2009) or semiconductor diodes (Waldhäusl et al 2005). TLDs involve offline process providing the integral dose absorbed during patient treatment and require special procedures in order to achieve good precision of the results. On the other hand, diodes show rapid processing time, high sensitivity and immediate reuse, however, they show a high energy dependence and the delivered dose is therefore not promptly inferred from the diode reading. Moreover, the major disadvantages of diodes are their relative large sizes, which make them unable to be held in many catheters placed inside the patient to perform in vivo dosimetry.

New detectors such as fiber optic coupled scintillation dosimeters (Suchowerska et al 2007, Therriault-Proulx et al 2011) and metal oxide semiconductor field effect transistors (MOSFETs) (Zilio et al 2006, Fagerstrom et al 2008) have recently been introduced to perform in vivo dosimetry. In particular, MOSFETs show many advantages, such as good spatial resolution, high 
sensitivity, real time read-out without deterioration of information, negligible radiation field perturbation owing to their small size and ease of use. In particular, great interest was dedicated to the application of MOSFETs to BT, because the typical large dose gradients achieved in BT necessitate a small detector with a reduced active volume for accurate dosimetry. In this work, a specific type of MOSFET dosimeter called "MOSkin" which has developed by the Centre for Medical Radiation Physics (CMRP) of the University of Wollongong (Australia) (Qi et al 2007, Kwan et al 2008, Kwan et al 2009) has been studied.

\subsection{High dose rate prostate brachytherapy}

High dose rate (HDR) prostate BT allows the delivery of local and high conformal dose directly into the tumour, minimizing exposure of the surrounding healthy tissues. Due to the large dose delivered to the target in a single fraction and the dose constraints to be simultaneously satisfied for organs at risk, it is very important to have as small as possible discrepancy between planned and delivered dose. The development and application of reliable and accurate methods for monitoring the dose delivered to critical organs is therefore crucial.

Among these organs at risk, the urethra is most likely susceptible to acute and/or late toxicity resulting from the treatment (i.e. urethritis, stenosis), as it is inside the target volume (figure 1a). However, its localization for treatment planning purposes is particularly difficult due to images artefacts generated by the presence of source catheters, especially if transrectal ultrasound imaging is performed. Moreover, source catheters are themselves difficult to be accurately localized on the same images and therefore calculated dose distributions are susceptible to inaccuracies (figure 1b). The real time dosimetry in the urethra is therefore very important and will be supplementary to reinforce existing QA programs.

Studies aimed at characterizing the dosimetric properties of MOSkin dosimeters have already demonstrated that they are promising instruments for performing in vivo dosimetry during HDR BT treatments (Qi et al 2007, Kwan et al 2008, Qi et al 2012). Measurements finalized to detect the 
accuracy of the dosimeters and the change in sensitivity as a function of depth and angle of incidence of the radiation, have already shown good agreement between MOSkin response and dose calculated by the TPS (Hardcastle et al 2010). Aim of this work was to study and develop the applicability of the MOSkin dosimeters for urethral dose measurement in prostate HDR BT.

\section{Materials and Method}

\subsection{MOSkin dosimetry system}

The design of this particular type of MOSFET is optimized to measure dose in steep dose gradients. Different from other commercial MOSFETs, MOSkin die is embedded in a thin kapton layer and hermetically sealed with water-equivalent flexible carrier of reproducible thickness and avoid traditional wire bonding with high $-\mathrm{Z}$ wires . The sensitive volume, defined by the volume of the gate oxide, is $4.8 \times 10^{-}$

${ }^{6} \mathrm{~mm}^{3}$. MOSkin detectors can be adopted alone or coupled in a face-to-face arrangement. This face-to-face dual-MOSkin arrangement is referred to in this text as the "dual-MOSkin". The dual-MOSkin, proposed and developed at CMRP, allows for angular-independent measurements as it compensates the naturally asymmetrical structure of the MOSFET chip relative to the beam direction (Hardcastle et al 2010). The dosimetry system adopted with MOSkin detectors includes a microprocessor based reader which is connected to a laptop provided with a dedicated software ("MosPlot"). The computer data acquisition system measures periodically the instantaneous voltage signal with a user defined frequency. The "MosPlot" software allows for the online graphical representation of the change in the threshold voltage (proportional to accumulated dose) or increments in the threshold voltage for consecutive readouts (proportional to dose rate).

\subsection{Nucletron Microselectron-HDR Brachytherapy facility}

BT irradiations were performed using a Microselectron-HDR (Nucletron,Veenendaal, the

Netherlands) high dose rate remote afterloading device. The facility is provided with a ${ }^{192}$ Ir 
radioactive source which has an active length of $3.6 \mathrm{~mm}$ and a diameter of $0.65 \mathrm{~mm}$. The source is sealed inside a capsule that is welded to one end of a flexible steel cable and the treatment unit moves it to the required dwell positions.

Experimental determinations of absolute dose rates to water from high-energy sources should have direct traceability of the source Air Kerma Strength Sk to a primary or secondary standard dosimetry laboratory (PSDL OR SSDL) (Perez-Calatayud et al, 2012). In our case, the $\mathrm{S}_{\mathrm{k}}$ was certificated by the vendor with $5 \%(\mathrm{k}=3)$ uncertainty using a calibration instrument traceable to the standard of the PSDL Physikalisch-Technische Bundesanstalt (PTB, Braunschweig, Germany). Moreover, the certified $S_{\mathrm{k}}$ was independently verified at our hospital before its clinical application, by the qualified medical physicist using a calibrated well-type chamber traceable to a PSDL. The well-type chamber has a vented sensitive volume of 200cc (Source Dosimetry System. Nucletron, Veenendaal, the Netherlands) (Nath et al 1997) and has proven to be a fast and precise measuring instrument (Azhari et al, 2012). The delivered dose distribution is calculated by TPS Oncentra Brachytherapy (Nucletron, Veenendaal, the Netherlands) and is based on the recommendations of the American Association of Physics in Medicine (AAPM) TG-43 report (Rivard et al 2004)..

\subsection{MOSkin and dual-MOSkin calibration and response at increasing source-detector distance}

Each single MOSkin and dual-MOSkin was calibrated in a water phantom at a distance of $38 \mathrm{~mm}$

from the ${ }^{192}$ Ir HDR source. A suitable support for accurate and reproducible detector and source positioning was developed to perform this task (figure 2) and the experimental set-up was accurately defined by means of CT imaging (slice thickness: $0.8 \mathrm{~mm}$ ) and catheter digital reconstruction. Five irradiations were performed for defined source dwell times and the mean values of MOSkin threshold voltage changes were obtained. Each detector was finally calibrated considering the correspondent dose value calculated by means of the TPS in the same position where the active component of the dosimeter was placed. To investigate MOSkin sensitivity variation vs source-detector distance due to changing of the photon energy spectrum from the Ir- 
192 source, distance dependent correction factor (CF) was evaluated for measurements performed at locations other than the calibration position, ranging from $7 \mathrm{~mm}$ to $46 \mathrm{~mm}$ and from $11 \mathrm{~mm}$ to $44 \mathrm{~mm}$ along the source transverse axis in the water phantom for MOSkin and dual-MOSkin dosimeters, respectively. In each one of these locations, measurements were repeated five times.

\subsection{In phantom simulations of prostate treatments}

With the aim of performing dose measurements with implants and conditions simulating typical prostate treatments, a suitable gel phantom was realized. The phantom has a cylindrical shape with length of $14.5 \mathrm{~cm}$, and a diameter of $17.6 \mathrm{~cm}$. The urethral catheter and 14 interstitial needles were placed inside the gel phantom through a real prostate treatment template. Single or dual-MOSkins were inserted in the urethral catheter. CT images $(0.8 \mathrm{~mm}$ slice thickness $)$ were acquired in order to precisely localize needles and the urethral catheter containing the dosimeter. In the treatment planning phase, the presence of a prostate was virtually created by drawing it on the CT images around the needles implant. The urethral catheter was as well segmented and different treatments were planned by prescribing the dose to the outlined prostate. The expected doses to the dosimeters were evaluated from the TPS. In fact, MOSkin detectors could be accurately recognized on CT images' reconstructions as small radiopaque spots inside the urethral catheter (figure 3). During irradiation, the plastic needles were connected to the BT afterloader system thought transfer tubes and MOSkins were connected to the CMRP computerized reader for the on-line acquisition of dose measurements (figure 4).

A series of measurements of the same irradiation set-up was performed with different dose prescriptions according to the developed treatment plans. The total urethral dose measured with the single or dual-MOSkin detector was compared to the expected dose in the same point. Moreover, the contributions to the total dose given by the source moving in each single needle were calculated separately and compared to the measured ones. 


\section{Results and discussion}

Single MOSkin and dual-MOSkin detectors calibration factors obtained in the water phantom on the Ir-192 source resulted $0.38 \mathrm{cGy} / \mathrm{mV}$ and $0.30 \mathrm{cGy} / \mathrm{mV}$, respectively. Distance dependent CFs at different source-detector distances for single MOSkin data were in accordance with those reported in Qi et al (2012); the significant increase of CF at decreasing source-detector distance was observed. In contrary to single MOSkins, CFs for dual-MOSkins showed a relatively flat response with changing the Ir-192 source-detector distance (figure 5). It is important to note that reference dose data to perform dosimeter calibration were not obtained experimentally by a further dosimeter, but were those resulting from the TPS and cross-checked with a home made software based on the AAPM TG-43 algorithm (Rivard et al, 2004). The chosen $\Lambda, G(r, \theta), F(r, \theta)$ and $g(r)$ factors were those tabulated in Daskalov et al (1998), which were obtained by means of a Monte Carlo (MC) photon transport code knowing the characteristics of the Nucletron model mHDR-v2 source. Consensus on these data has been widely achieved and the resulting AAPM TG-43 algorithm calculations, when performed in reference conditions of unbounded water medium, are highly accurate (Daskalov et al, 1998). In fact, the role of experimental dosimetry for high-energy BT may be more limited than MC-based dosimetry. With high energy BT sources, range and spatial resolution limitations are for instance not of concern for MC methods (Perez-Calatayud et al, 2012).

Comparison of total dose measured in the gel phantom for the BT prostate implant with the singleMOSkin detector and the expected doses in the same points are reported in Table 1. Measurements were repeated for three different treatment plans with different dose prescriptions. The maximum discrepancy between expected and measured dose was found to be $8.9 \%$. Figure 6a shows measured and calculated contribution to the total dose resulting by the source dwelling in each of the 14 needles adopted to simulate the prostate implant, for one of the three different sets of measures. 
Excluding needles that give a low contribution to the cumulative dose $(<20 \mathrm{cGy})$, single point dose differences resulted less than $15 \%$.

Comparison of total doses measured with the dual-MOSkin detector and the expected doses in the same point is reported in Table 2 for three different measurement sessions. It was found that discrepancy was within $3.8 \%$. Figure $6 \mathrm{~b}$ shows measured and calculated contribution to the total dose resulting by the source moving in each of the 14 needles adopted to simulate the prostate implant, for one of the measured treatment sessions. Excluding needles that give a low contribution to the cumulative dose $(<20 \mathrm{cGy})$, single dose differences resulted in general smaller then those resulting with a single MOSkin readings (i.e. $\max 8 \%$ ).

Results obtained for both single MOSkin and dual-MOSkin dosimeters are summarized in the plot in figure 7. Dual-MOSkin configuration provides greater accuracy than a single MOSkin detector as angular isotropy and depth dose are greatly improved. Single MOSkin accuracy could be partially increased by applying measured CFs at changing source-detector distances and further CFs that take into account its orientation with respect to the source position. However, CFs are almost impossible to be correctly adopted whenever MOSkins are used as real-time in vivo dosimeters because sourcedetector distances and MOSkin orientation cannot be easily quantified and taken into account.

\section{Conclusions}

In prostate HDR BT treatments, a very high dose over a few fractions is delivered. In such conditions, real time dosimetry is most valuable in detecting a dose error at the onset of treatment, providing the capability of immediate corrections. Measurements performed in a phantom simulating a typical prostate implant with MOSkin detectors placed within the urethral catheter have shown that they allow evaluation of the actual dose to the urethra during a treatment fraction. Through an online comparison between the measured and expected dose, the dosimetry system 
might offer a further security to detect and prevent significant errors in the clinical routine of HDR BT prostate treatments.

\section{Acknowledgments}

This work was partially supported by the National Institute of Nuclear Physics (INFN), Italy, and by the "5 per mille" project of the Italian Government.

\section{References}

Azhari HA, Hensley F, Schütte W, Zakaria GA. 2012. Dosimetric verification of source strength for HDR afterloading units with Ir-192 and Co-60 photon sources: Comparison of three different international protocols. J Med Phys. 374, 183-92.

Daskalov G.M., Löffler E., Williamson J.F. 1998. Monte Carlo-aided dosimetry of a new high dose-rate brachytherapy source. Med. Phys. 25, 2200-2208.

Fagerstrom J.M., Micka J.A., DeWerd L.A. 2008. Response of an implantable MOSFET dosimeter to 192-Ir HDR radiation. Med. Phys. 35, 5729-37.

Hardcastle N., Cutajar D., Metcalfe P.E., Lerch M., Perevertaylo V.L., Tomé W.A., Rosenfeld A.B., 2010. In vivo real-time rectal wall dosimetry for prostate radiotherapy, Phys. Med. Biol. 55, 3859-3871.

Kwan I.S., Rosenfeld A.B., and Qi Z.Y., 2008. Skin dosimetry with the new MOSFET detectors, Rad. Meas. 43, 929-932.

Kwan I.S., Wilkinson D., Cutajar D., Lerch M., Wong Y., Bucci J., Perevertaylo V., Rosenfeld A.B. 2009. The effect of rectal heterogeneity on wall dose in High Dose-Rate brachytherapy, Med. Phys. 36, 224-232. 
Lambert J., Nakano T., Law S., Elsey J.,. McKenzie D.R, Suchowerska N. 2007. In vivo dosimeters for HDR brachytherapy: A comparison of a diamond detector, MOSFET, TLD, and scintillation detector. Med. Phys. 34, 1759-65.

Mijnheer B. 2008. State of the art of in vivo dosimetry. Radiat. Prot. Dosim. 131, 117-22.

Nath R., Anderson L.L., Meli J.A., Olch A.J., Stitt J.A., Williamson J.F. 1997. Code of practice for brachytherapy physics: Report of the AAPM Radiation Therapy Committee Task Group No.56. Med. Phys. 24, 1557-1598.

Perez-Calatayud J, Ballester F, Das RK, Dewerd LA, Ibbott GS, Meigooni AS, Ouhib Z, Rivard MJ, Sloboda RS, Williamson JF. 2012 Dose calculation for photon-emitting brachytherapy sources with average energy higher than $50 \square \mathrm{keV}$ : report of the AAPM and ESTRO. Med Phys. 39, 290429.

Qi Z.Y., Deng X.W., Huang S.M., Jie L., Lerch M., Cutajar D., Rosenfeld A.B. 2007. Verification of the plan dosimetry for high dose rate brachytherapy using MOSFET detectors. Med. Phys. 34, 2007-2013. 
Qi Z.Y., Deng X.W., Cao X.P., Huang S.M., Lerch M. and Rosenfeld A. 2012. A real-time in vivo dosimetric verification method for high dose rate intracavitary brachytherapy of nasopharyngeal carcinoma. Med. Phys. 39, 6756-63.

Rivard M.J., Coursey B.M., DeWerd L.A., Hanson W.F., Huq M.S., Ibbott G.S., Mitch M.G., Nath R., Williamson J.F. 2004. Update of AAPM Task Group No. 43 Report: A revised AAPM protocol for brachytherapy dose calculations. Med. Phys. 31, 633-674.

Suchowerska N., Lambert J., Nakano T., Law S., Elsey J., McKenzie D.R. 2007. A fibre optic dosimeter customised for brachytherapy. Radiat. Meas. 42, 929-32.

Therriault-Proulx F., Briere T.M., Mourtada F., Aubin S., Beddar S., Beaulieu L., 2011. A phantom study of an in vivo dosimetry system using plastic scintillation detectors for real time verification of 192Ir HDR brachytherapy. Med. Phys. 38, 2542-51.

Toye W., Das R., Kron T., Franich R., Johnston P., Duchesne G. 2009. An in vivo investigative protocol for HDR prostate brachytherapy using urethral and rectal thermoluminescence dosimetry. Radiother. Oncol. 91, 243-8.

Waldhäusl C., Wambersie A., Pötter R., Georg D., 2005. In-vivo dosimetry for gynaecological brachytherapy: physical and clinical considerations. Radiother Oncol. 773, 310-7.

Zilio V., Joneja O., Popowski Y., Rosenfeld A., Chawla R., 2006 Absolute depthdose-rate measurements for an 192/r HDR brachytherapy source in water using MOSFET detectors. Med. Phys. 33, 1532-9. 


\section{Figure captions}

Figure 1: a) 3D graphical representation of an HDR brachytherapy plan of the prostate (dark red). Urethra, rectum and bladder are represented in yellow, green and blue, respectively. Source catheters are drawn as green lines, source dwell positions are represented by red spheres and resulting dose distribution (95\% isodose) is given in light blue; b) transversal prostate image with the resulting dose distribution. Characteristic hot spots are present around some of the implanted needles.

Figure 2: Support to be placed in a water phantom which was developed for dosimeters calibration. Source catheter can be placed at different distances from the dosimeter.

Figure 3: a) sagittal CT reconstruction and b) transversal CT image including the urethral catheter. MOSkin dosimeter is clearly recognizable as a radiopaque spot inside the catheter.

Figure 4: Experimental set-up used for in phantom measurements.

Figure 5: Correction factors evaluated at different source-detector distances for single- (white circles) and dual-MOSkin (gray diamonds) detectors. Plotted error bars are given by the standard deviation of the measurements and result in the values of $0.025,0.018,0.018,0.019,0.020,0.019$ and of $0.046,0.042,0.043,0.048,0.034,0.032$ for single- and dual-MOSkin, respectively.

Figure 6: Measured and expected doses given by the source moving in each of the 14 needles for a) MOSkin and b) dual-MOSkin detectors.

Figure 7: Comparison between single MOSkin and dual-MOSkin in terms of measured mean dose differences between expected and measured values, standard errors and standard deviations. 
Tables

Table 1: Total urethral dose measured with a single-MOSkin dosimeter for three different treatments.

\begin{tabular}{|c|c|c|c|}
\hline \multicolumn{3}{|c|}{ Total urethral dose } \\
\hline & $\begin{array}{c}\text { Measured Dose } \\
\text { (cGy) }\end{array}$ & $\begin{array}{c}\text { Calculated Dose } \\
(\mathbf{c G y})\end{array}$ & $\begin{array}{c}\text { Dose difference } \\
(\%)\end{array}$ \\
\hline $\mathbf{1}$ & 727.2 & 798.1 & -8.9 \\
\hline $\mathbf{2}$ & 809.2 & 828.1 & -2.0 \\
\hline $\mathbf{3}$ & 732.0 & 799.0 & -8.0 \\
\hline
\end{tabular}

Table 2: Total urethral dose measured with a dual-MOSkin dosimeter for three sessions of the same treatment.

\begin{tabular}{|c|c|c|c|}
\hline \multicolumn{4}{|c|}{ Total urethral dose } \\
\hline & $\begin{array}{c}\text { Measured Dose } \\
\text { (cGy) }\end{array}$ & $\begin{array}{c}\text { Calculated Dose } \\
\text { (cGy) }\end{array}$ & $\begin{array}{c}\text { Dose difference } \\
(\%)\end{array}$ \\
\hline $\mathbf{1}$ & 1139.5 & 1148.5 & -0.8 \\
\hline $\mathbf{2}$ & 1126.7 & 1148.5 & -1.9 \\
\hline $\mathbf{3}$ & 1104.3 & 1148.5 & -3.8 \\
\hline
\end{tabular}


Figures
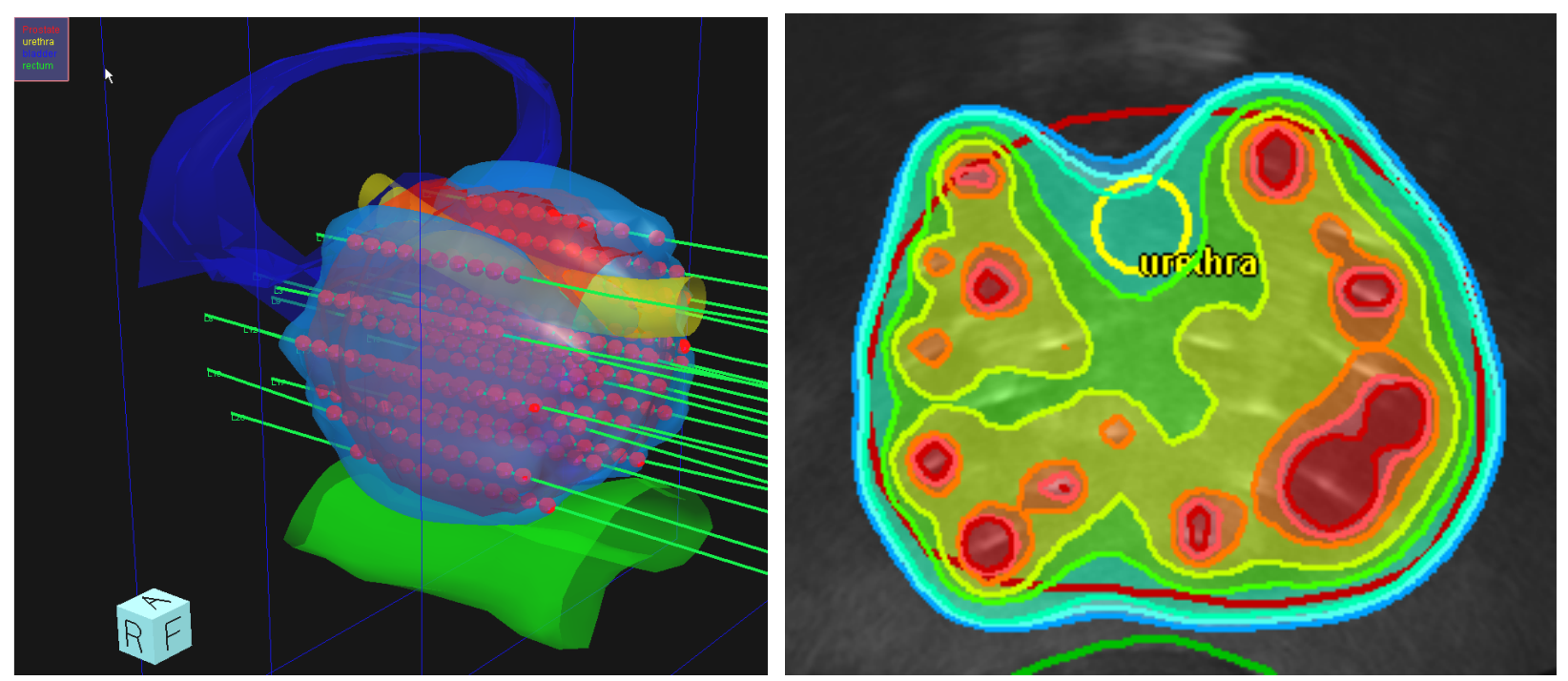

Figure 1 


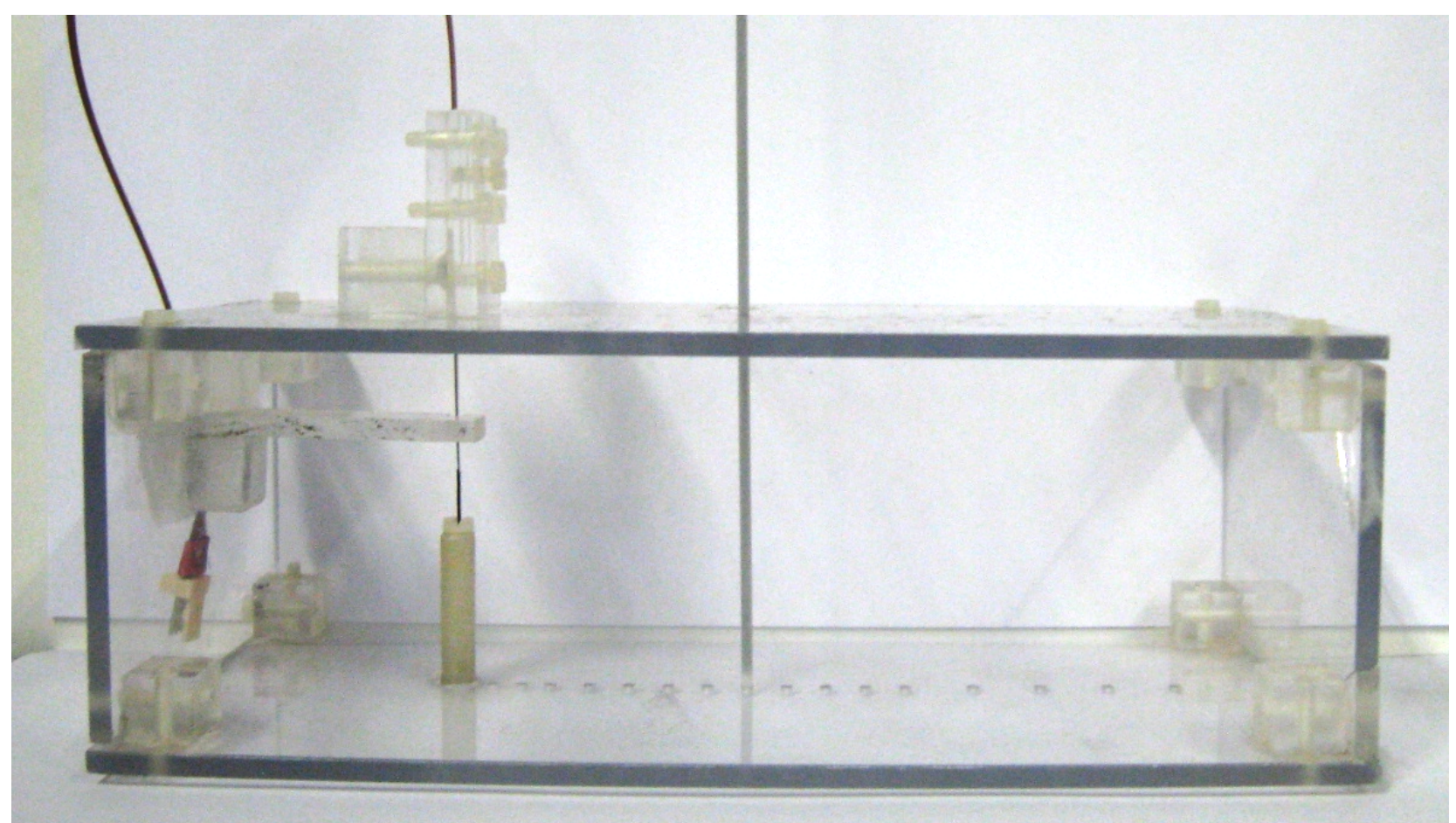

Figure 2 

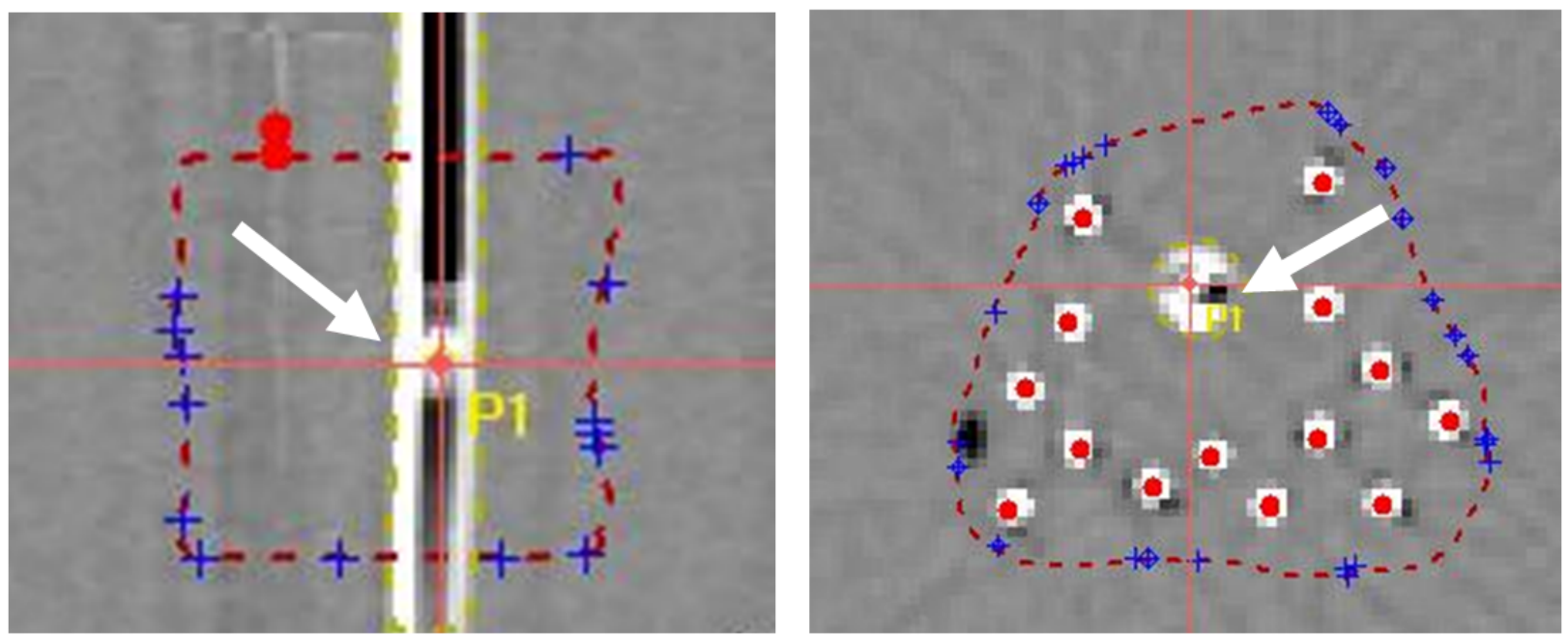

Figure 3 


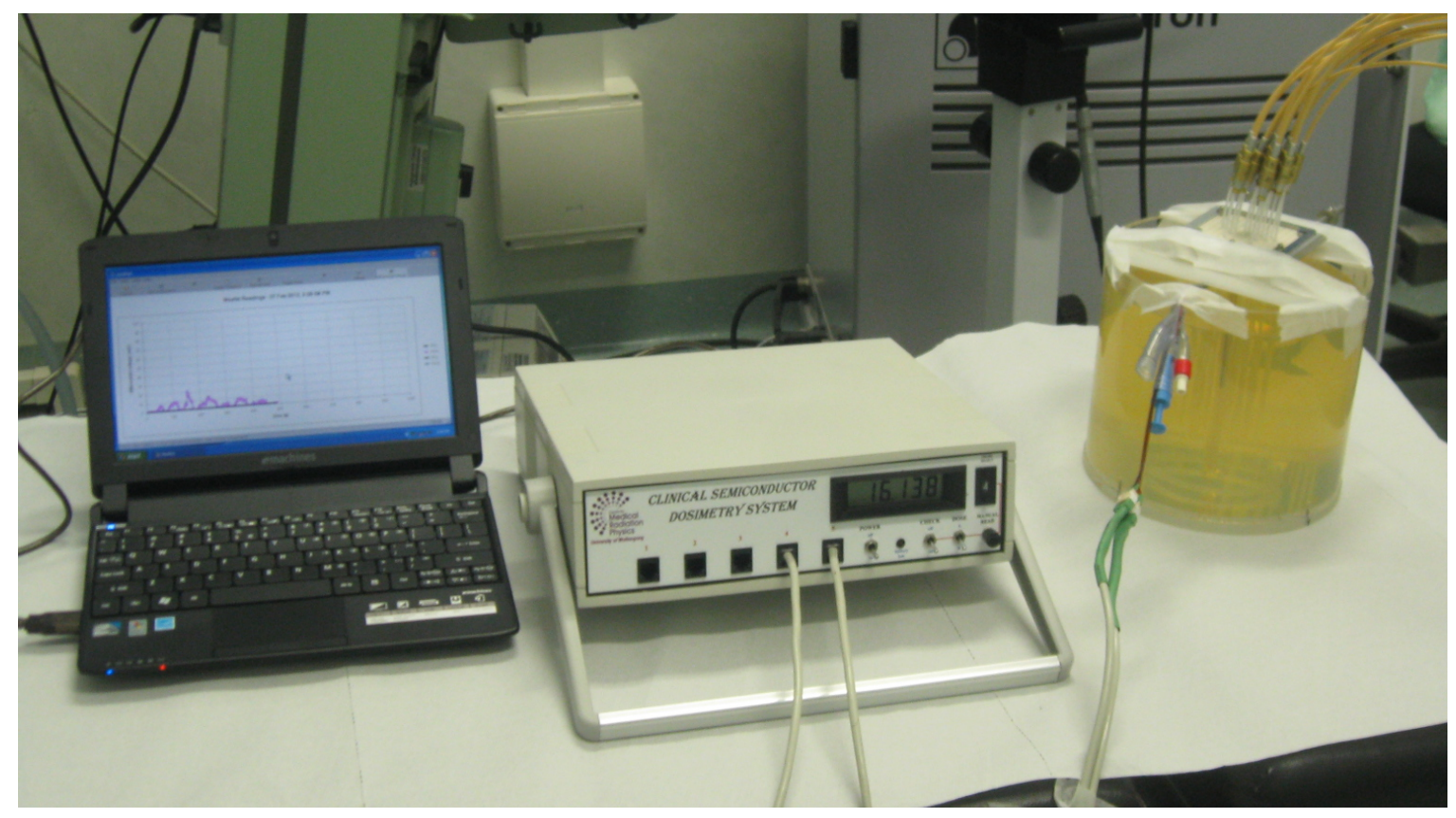

Figure 4 


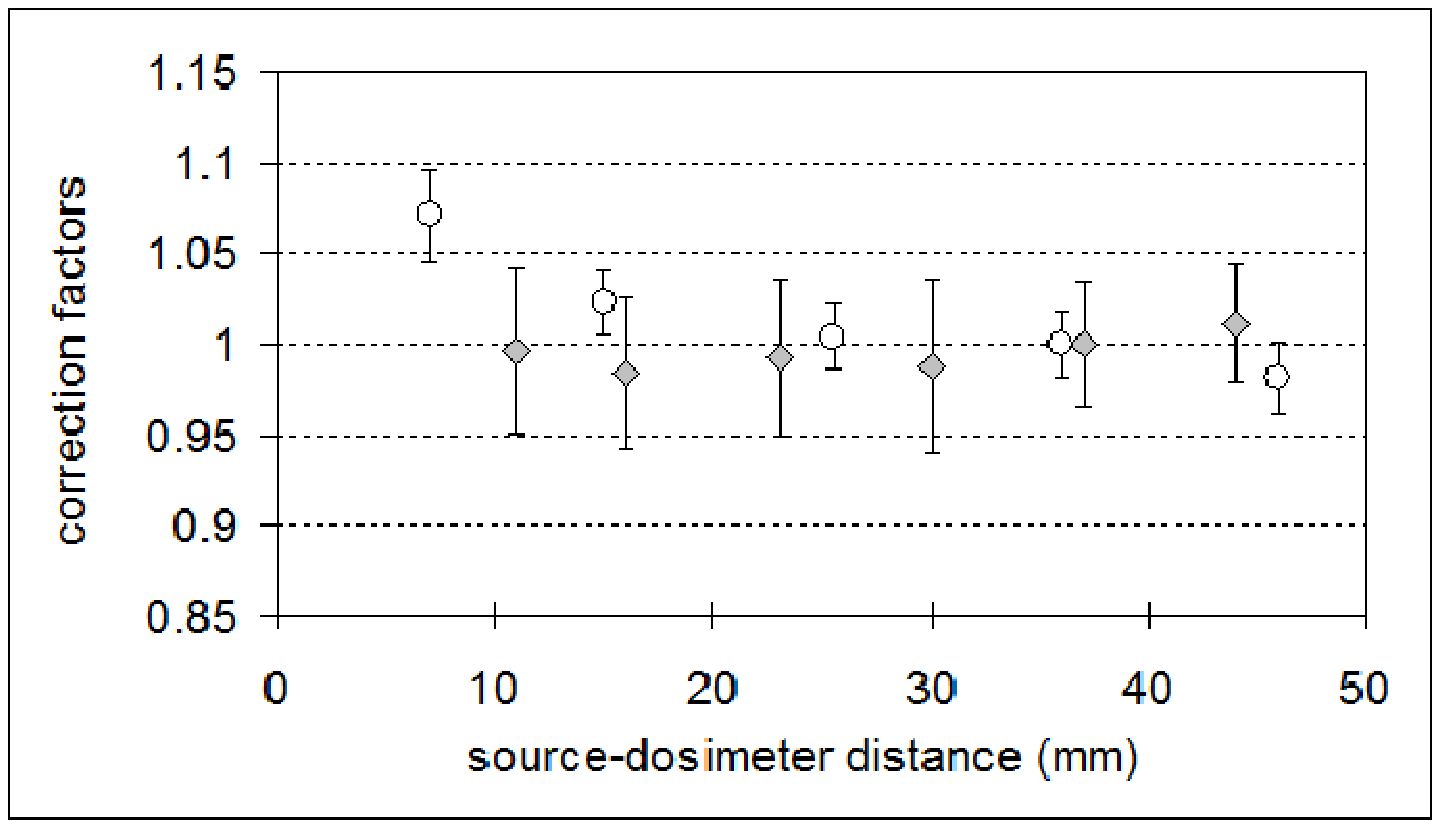

Figure 5 

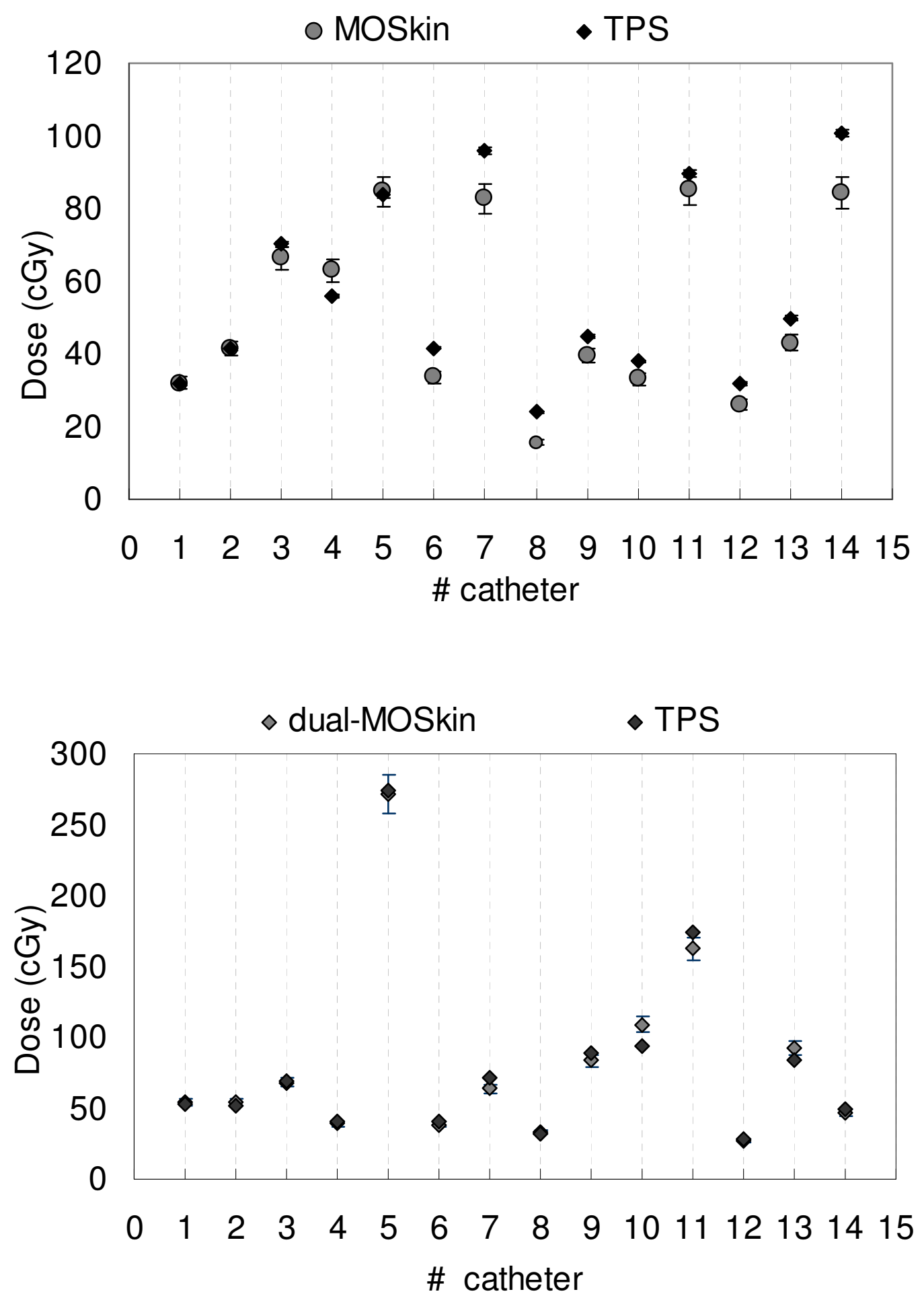

Figure 6 


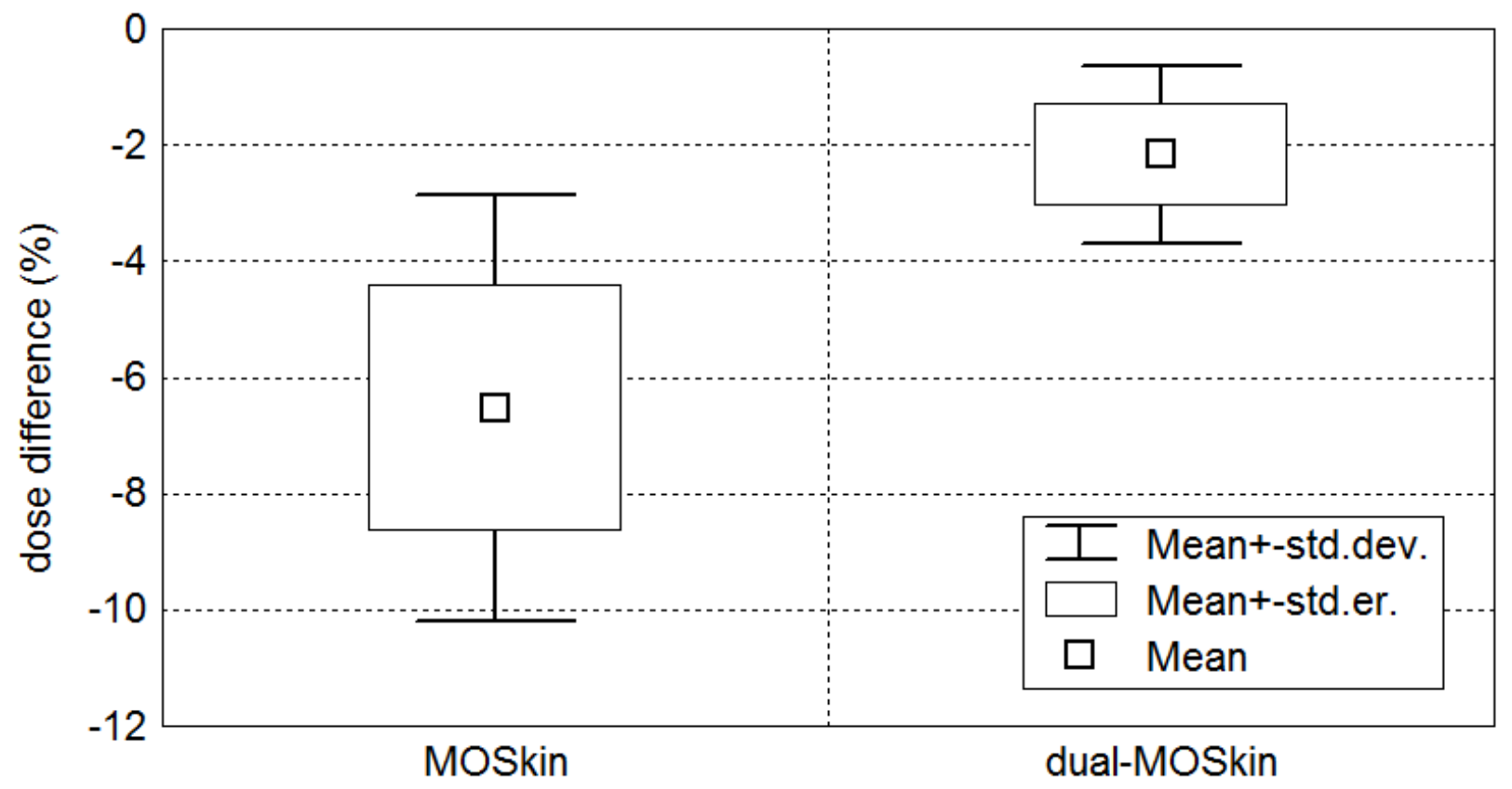

Figure 7 